\title{
Probably Benign Solid Breast Lesions on Ultrasound: Need for Biopsy Reassessed
}

\author{
Kulsoom Fatima*, Imrana Masroor, Samina Khanani
}

\begin{abstract}
Objective: To determine the negative predictive value of ultrasound for breast masses with probably benign morphology, and to assess whether follow-up may be an acceptable alternative to biopsy. Methods: After Institutional Review Board approval, all solid breast masses categorized as probably benign (American College of Radiology Breast Imaging Reporting and Data System [BI-RADS] 3) on ultrasound from January 2014 to December 2015, and having either tissue diagnosis or imaging stability for 24 months, or downgrading to BIRADS 2 during imaging surveillance were included. Result: A total of 157 lesions in 40 patients constituted the study population. The mean patient age was 31.3 years (range, 20-56 years). Seventeen of these 157 lesions underwent tissue diagnosis with no invasive breast cancer. Out of the remaining 140 lesions, 115 were stable on imaging for 24 months or more. The rest 25 were deemed benign because of decrease in size on follow up $(n=1)$, non-recommendation of further imaging by the second radiologist on follow up ultrasound $(n=13)$ or presence of benign tissue diagnosis in the largest lesion $(n=11)$. Conclusion: Ultrasound has $100 \%$ negative predictive value for breast lesions with probably benign morphology, whether palpable or not. Follow up is an appropriate option to immediate biopsy of such lesions keeping in mind that noncompliance with surveillance may be a potential problem.
\end{abstract}

Keywords: BI-RADS- probably benign- biopsy- ultrasound- palpable- non palpable

Asian Pac J Cancer Prev, 19 (12), 3467-3471

\section{Introduction}

Sonography, in addition to mammography, has become a standard breast imaging tool in the past couple of decades because of significant improvements in equipment as well as clinical experience resulting in improved accuracy of assessing breast lesions (Costantini et al., 2006; Giess et al., 2012).

It is widely available, easily tolerated and free from adverse effects of radiation or contrast medium (Chae et al., 2016).

Earlier, ultrasound was predominantly employed in differentiating between cystic and solid breast masses however its role has now expanded from simply characterizing the internal contents of the mass to differentiating between benign and malignant solid masses. It may also be the screening tool for young women or those with dense breasts (Costantini et al., 2006; Kim et al., 2008; Shin et al., 2015) and an important adjunct to mammography in older patients as well as a primary imaging tool for women younger than 40 years who present with a palpable breast mass (Constance et al., 2014).

As a result, the Breast Imaging Reporting and Data System (BI-RADS) lexicon, initially developed for mammography was expanded to include a breast ultrasound lexicon. The American College of Radiology released the lexicon for breast US in 2003. This not only provides and facilitates better communication between the radiologists and clinicians but also guarantees uniformity to recommendations (ACR, 2003).

The abnormalities detected on ultrasound are categorized into six categories. A lump which corresponds to a clear benign finding, that is BIRADS 2 on ultrasound, such as a simple cyst, non-pathologic lymph node, lipoma, sebaceous cyst, clustered microcysts, or duct ectasia, can safely undergo clinical follow-up without short-interval imaging follow-up, needle aspiration, or biopsy.

The lesions categorized as BIRADS 3 are recommended an initial short follow up at 6 months followed by 12 months and 24 months follow up to establish stability.

Several studies have shown that an oval or gently lobulated shape, circumscribed margin, and parallel orientation on ultrasonography (US) are useful features for differentiating benign solid masses from malignant lesions, and that it is reasonable to categorize lesions with these characteristics as BI-RADS 3, probably benign (Stavros et al., 1995; Rahbar et al., 1999; Graf et al., 2004; Hong et al, 2005; Costantini et al., 2006; Graf et al., 2007; Chala et al., 2007; Leung and Sickles 2007; Kim et al., 2008).

The ACR BI-RADS manual 2003, 4th edition, 
recommended short-term follow-up only for non-palpable findings however as the rates of malignancy in palpable probably benign lesions are not significantly different from those of non-palpable probably benign lesions (Harvey et al., 2009; Shin et al., 2015), the current ACR manual, 2013, 5th edition supports the same recommendation for both (D'Orsi et al., 2013).

The malignancy rate of probably benign breast lesions on US ranges between $0 \%$ and $0.8 \%$ (Raza et al., 2008; Moon et al., 2010).

These results support the rationale that a short interval follow-up is an acceptable alternative to biopsy.

Despite many studies recommending follow up to be safe for BI-RADS 3 solid lesions, the practice at our institute is inconsistent. The contributing factors may be the level of experience or expertise of the radiologist and the confidence of the referring surgeon on her along with discrepancy in the clinical features. Our hospital is the largest private tertiary care center in the country and many patients seeking consultation come from outside the city. As there is a fear that they might not return for follow up and also to relieve their anxiety biopsy is often performed on BIRADS 3 lesions. This however has significant financial implications in our set up as the patient pays for the biopsy out of her own pocket. The outcome of such patients as well as those returning for follow up has not been previously assessed locally. This formed the rationale for performing this study. We intend to examine the negative predictive value of US for breast masses with probably benign morphology and to assess whether follow-up US may be an acceptable alternative to immediate biopsy.

\section{Materials and Methods}

\section{Study design and inclusion criteria}

The study was conducted at the Radiology Department of Aga Khan University Hospital, Karachi. This retrospective study was approved by the institutional review board and informed consent was waived. Ultrasound report of all consecutive patients undergoing breast ultrasound examination from January 2014 to December 2015 were reviewed and those patients who had initial breast ultrasound in this period categorized as BI-RADS 3 according to ACR ultrasound BIRADS lexicon were identified (Figure 1). These were 141 in number. Out of these, 101 patients were excluded due to either inadequate follow up $(n=62)$, appeared as cysts/duct on follow up ultrasound $(\mathrm{n}=12)$, had features suggesting mastitis/abscess $(n=21)$, intramammary lymph nodes $(n=3)$, galactocele $(n=1)$ and scar/seroma $(n=2)$. The remaining 40 patients with 157 masses constituted the study population (mean age, 31.3 years; age range, 20-56 years). These included patients/lesions with adequate follow up for 2 years, less than 2 years follow up with downgrading to BIRADS 2 category or those who underwent biopsy with histopathological confirmation.

\section{Imaging and interpretation}

Two radiologists, with experience of 10 and 13 years respectively in breast imaging, reviewed the sonographic images and reports and extracted patient's demographics. Lesions included in imaging surveillance were considered benign if remained stable for at least 24 months, were decreased in size on follow up imaging or had a benign tissue diagnosis in the larger lesion in cases where there were multiple lesions in the same patient. Sonographic examination was performed on Aplio 400 and Zario 200 Ultrasound machines with 7.5 $\mathrm{MHz}$ linear array transducer. All sonographic examinations were performed or supervised by attending radiologists with 5-15 years of experience in breast imaging.

The US categorization was based on ACR BI-RADS (Table 1) and the criteria established in the study by Stavros et al., (1995). The features on the basis of which the masses were categorized as BI-RADS 3 included oval or gently lobulated shape, circumscribed margins, long axis parallel to the skin, predominantly hypoechoic homogeneous internal echotexture, and no overt features of malignancy which are: irregular shape, microlobulations, angular or spiculated margins, and heterogeneous echotexture with posterior acoustic shadowing.

The number of lesions, side, location (quadrant), and maximum diameter was recorded.

Standard imaging surveillance protocol for probably benign lesion was followed which includes 6 months, 12 months and 24 months. The duration of follow up ranged from 6 to 36 months.

\section{Biopsy}

Percutaneous biopsy was performed in 17 lesions due to either change on imaging surveillance or physician's preference. None of the patient underwent FNA. Core biopsies were performed with 14- gauge needle using automated gun and four to six cores were obtained to ensure adequacy of the sample.

\section{Statistical analysis}

Statistical analysis was performed using SPSS statistical software version 22 for Windows.

False negative rate and negative predictive value (NPV) were calculated.

\section{Results}

A total of 157 lesions in 40 patients constituted the study population. The mean patient age was 31.3 years (range, 20-56 years). Out of these, 157 lesions 138 (88\%) occurred in women less than 40 years and 19 lesions (12\%) in patients 40 years or above (Table 2).

The mean lesion diameter was $13.4 \mathrm{~mm}$ (range, 3-46 mm). Majority of the lesions, 125 (79\%) were non palpable. Slightly more lesions, 81 (51.6\%) occurred in the right breast.

Based on location, retro areolar region was the least common site of involvement, 4 out of 157 (2.5\%) while upper outer quadrant the most common site of involvement, seen in 60 (38.2\%) lesions.

One hundred and sixteen lesions (74\%) were oval in shape while the rest were non oval.

About $50 \%$ of the lesions showed lobulations. Majority of the lesions, 117 (75\%) lesions had 
Table 1. American College of Radiology BIRADS Classification; Final Assessment Categories
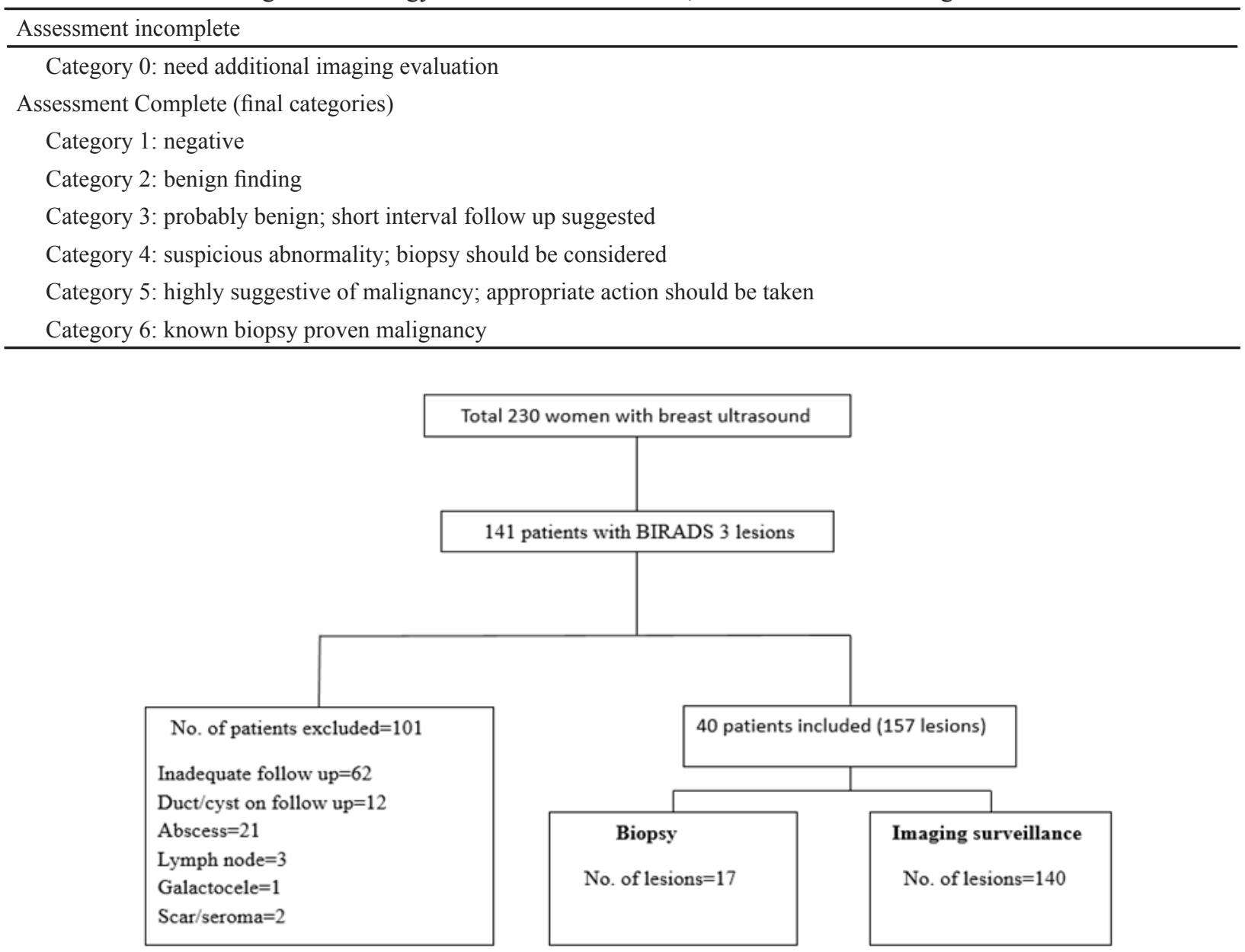

Figure 1. Flow Chart of the Study Population

a homogeneous echotexture. Most of the lesions 147 $(94 \%)$ showed a parallel orientation to the skin.

Seventeen out of total 157 lesions underwent tissue diagnosis with no invasive breast cancer (Figure 2). Out of these 17 lesions, 5 had shown increase on follow up imaging while the rest 12 were biopsied on surgeon's discretion.

The histopathology was benign fibro epithelial lesion in 16 patients and stromal sclerosis in 1 patient.

115 lesions out of remaining 140 were stable for 24 or more months and were considered benign (Figure 3 ).

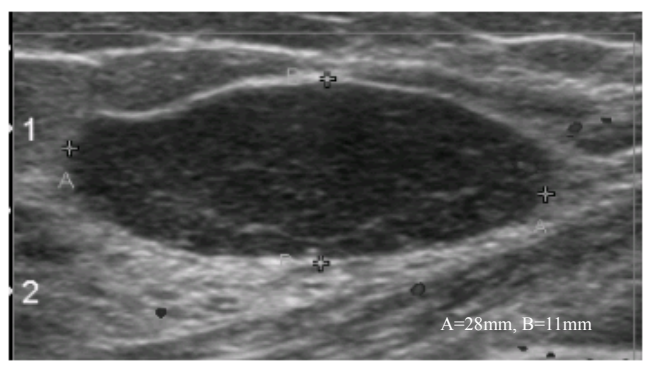

Figure 2. A 21-year-old Woman with a Palpable Mass in the Upper Inner Quadrant of Right Breast. Ultrasound shows a $28 \mathrm{~mm}$, oval, circumscribed, hypoechoic mass categorized as probably benign. Fibroadenoma was diagnosed by ultrasound-guided 14-gauge core needle biopsy.
Twenty five lesions had follow-up surveillance for less than 24 months (range; 6 months to 23 months). Out of these 25 lesions, eleven lesions occurred in those patients who had multiple lesions and the largest had already been proved benign on biopsy. One of the lesions

Table 2. Demographics of the Study Population and the Lesion Characteristics

\begin{tabular}{lc}
\hline Parameter & Number of lesions \\
\hline Age & 138 \\
$<40$ years & 19 \\
$>40$ years & \\
Multiplicity & 8 \\
$\quad$ Single & 149 \\
$\quad$ Multiple & \\
Palpability & 32 \\
Palpable & 125 \\
Non palpable & \\
Side involved & 81 \\
Right & 76 \\
Left & \\
Lesion size & \\
$<10 \mathrm{~mm}$ & 62 \\
$>10 \mathrm{~mm}$ & 95 \\
\hline
\end{tabular}




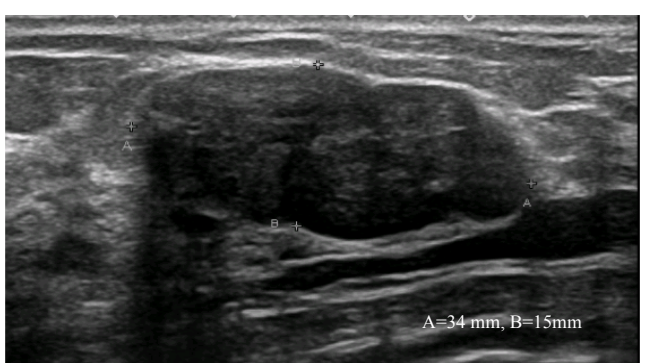

Figure 3. A 34-year-old Woman with a Palpable Lump in Right Breast. A $34 \mathrm{~mm}$ circumscribed, gently lobulated solid hypoechoic mass parallel to the skin categorized as probably benign on ultrasound. The lesion remained unchanged for 24 months.

decreased in size on follow up and hence was deemed benign. The reason for shorter follow up in remaining 13 lesions was that the repeat ultrasound was done by a different radiologist who based on her assessment did not recommend continued imaging.

\section{Discussion}

Ultrasound has proved to be a useful tool for evaluation of both palpable and non-palpable breast masses as it not only differentiates between solid and cystic lesions but also helps in characterizing the solid breast masses. However as the ultrasound is operator dependent, the interpretation as well as use of BI-RADS 3 category recommendation is variable. This is also true for follow up ultrasound as the second examiner may have different level of training and experience along with challenging technical factors such as breast size and lesion position which may alter the interpretation and hence assessment category (Raza et al., 2008). Thirteen of the lesions in our study were downgraded to BIRADS 2 category by the radiologist performing the follow up ultrasound. A very good agreement has been reported for lesion location and size while the margin assessment, echogenicity, and final assessment have lower agreement (Berg et al., 2006; Lazarus et al., 2006).

Despite these variations, the benign sonographic features have been reported to have high negative predictive value for malignancy ranging from 96 to 100\% (Stavros et al., 1995; Skanne and Engedal, 1998; Paulinelli et al., 2005; Mainiero et al., 2005; Graf et al., 2007; Kim et al., 2008). Some of these studies have been done on palpable masses while some included only non-palpable lesions. The palpability of a mass does not always indicate a new development or a growth in the lesion. The lesion may be palpable due to its superficial nature or may catch patient attention secondary to newly initiated breast examination and sometimes even due to trauma. Our study included patients with both palpable and non-palpable lesions however the majority of the lesions were non-palpable (79\%). The reason for less number of palpable lesions could be the fact the reporting radiologists may have a variable approach to palpable solid lesions, some recommending tissue diagnosis rather than surveillance and categorizing them as BIRADS 4 lesions regardless of benign imaging features. None of the biopsied lesion in our study showed malignancy, all were benign, with $94 \%$ being fibroadenomas. Among the patients undergoing imaging surveillance, all were stable and did not show any overt features of malignancy later during follow up. Hence our results showed 100\% negative predictive value of ultrasound for breast lesions categorized as BI-RADS 3.

These results are comparable to the study done by Patterson et al (2014) which showed no cancer in BIRADS 3 group on biopsy and the lesions showed stability on follow up imaging. The study done by Raza et al., (2008), although did show cancer in the BIRADS 3 masses, the number was very small $(0.8 \%)$ and all were diagnosed on initial 6-month follow-up examination, also they were less than $1 \mathrm{~cm}$ in size and without positive axillary nodes. The minimal delay in diagnosis had no detrimental effect on the patients. Park et al., (2008) also showed a very high negative predictive value $(99.4 \%)$ of ultrasound in BIRADS 3 lesions.

A factor to keep in consideration regarding follow up recommendation is patient compliance. A large of patients in our study were initially excluded (62 out of 141) as they failed to return for follow up and did not undergo the recommended imaging surveillance. Biopsy may therefore be a safe alternative in selected BI-RADS 3 cases where there is a strong likelihood that the patient might not return.

There were a few limitations in our study. A significant number of patients with BI-RADS 3 lesions did not come back for adequate follow up resulting in decrease in sample size. This may be because of cost issues as our hospital is a private tertiary care center and many patients do not have the resources to return for follow up and often continue care at their local facilities. Secondly, not all patients had complete 24 months follow up. In several of these patients the initial and follow up ultrasound examinations were performed by different examiners and the second examiner did not recommend continued imaging. This highlights the fact that assuring the same examiner for follow up may ensure adherence to the recommended imaging protocol for BI-RADS 3 lesions. Third, in patients with multiple lesions, since the largest lesion proved benign on histopathology the other smaller lesions were also deemed benign and follow up was discontinued.

To conclude, ultrasound has a very high negative predictive value for breast lesions with probably benign morphology. We therefore suggest follow up as an appropriate option to immediate biopsy of such lesions keeping in mind that non-compliance with surveillance may be a potential problem. A study with larger number of patients may further validate this study.

\section{Funding Statement}

No funding was provided for this manuscript.

\section{References}

American College of Radiology. Breast Imaging Reporting and Data System: BI-RADS Atlas. 4th ed. (2003) Reston, VA: 
American College of Radiology.

Berg WA, Blume JD, Cormack JB, Mendelson EB (2006). Operator dependence of physician performed whole-breast US : lesion detection and characterization. Radiology, 241, 355-65

Chae EY, Cha JH, Shin HJ, Choi WJ, Kim HH (2016). Reassessment and follow-up results of BI-RADS category 3 lesions detected on screening breast ultrasound. AJR, 206, 666-72

Chala L, Endo E, Kim S, et al (2007). Gray-scale sonography of solid breast masses: diagnosis of probably benign masses and reduction of the number of biopsies. J Clin Ultrasound, 35, 9-19

Costantini M, Belli P, Lombardi R, et al (2006). Characterization of solid breast masses: Use of the sonographic breast imaging reporting and data system lexicon. $J$ Ultrasound Med, 25, 649-59

D'Orsi CJ, Sickles EA, Mendelson EB, et al (2013). ACR BI-RADS atlas, breast imaging reporting and data system. Reston. Am Coll Radiol, 272, 80-5.

Giess CS, Smeglin LZ, Meyer JE, Ritner JA, Birdwell RL (2012). Risk of malignancy in palpable solid breast masses considered probably benign or low suspicion: Implications for management. J Ultrasound Med, 31, 1943-9

Graf O, Helbich TH, Fuchsjaeger MH, et al (2004). Follow-up of palpable circumscribed noncalcified solid breast masses at mammography and US: can biopsy be averted? Radiology, 233, 850-6

Graf O, Helbich TH, Hopf G, Graf C, Sickles EA (2007). Probably benign breast masses at US: is follow-up an acceptable alternative to biopsy?. Radiology, 244, 87- 93.

Harvey JA, Nicholson BT, Lorusso AP, Cohen MA, Bovbjerg VE (2009). Short-term follow-up of palpable breast lesions with benign imaging features: evaluation of 375 lesions in 320 women. AJR Am J Roentgenol, 193, 1723-30

Hong AS, Rosen EL, Soo MS, Baker JA (2005). BI-RADS for sonography: positive and negative predictive values of sonographic features. AJR Am J Roentgenol, 184, 1260-5

Kim EK, Ko KH, Oh KK, et al (2008). Clinical application of the BI-RADS final assessment to breast sonography in conjunction with mammography. AJR Am J Roentgenol, 190, 1209-15

Lehman CD, Lee AY, Lee CI (2014). Imaging Management of Palpable Breast Abnormalities. AJR Am J Roentgenol, 203, 1142-53

Leung JW, Sickles EA (2007). The probably benign assessment. Radiol Clin North Am, 45, 773-89.

Mainiero MB, Goldkamp A, Lazarus E, et al (2005). Characterization of breast masses with sonography: can biopsy of some solid masses be deferred?. J Ultrasound Med, 24, 161-7

Moon HJ, Kim MJ, Kwak JY, Kim EY (2010). Probably benign breast lesions on ultrasonography: A retrospective review of ultrasonographic features and clinical factors affecting the BI-RADS categorization. Acta Radiol, 51, 375-82.

Park YM, Kim EK, Lee JH, et al (2008). Palpable breast masses with probably benign morphology at sonography: can biopsy be deferred?. Acta Radiol, 49, 1104-11.

Patterson SK, Neal CH, Jeffries DO, et al (2014). Outcomes of solid palpable masses assessed as BI-RADS 3 or 4A: a retrospective review. Breast Cancer Res Treat, 147, 311-6.

Paulinelli RR, Freitas-Junior R, Moreira MAR, et al (2005). Risk of malignancy in solid breast nodules according to their sonographic features. J Ultrasound Med, 24, 635-41.

Rahbar G, Sie AC, Hansen GC, et al (1999). Benign versus malignant solid breast masses: US differentiation. Radiology, 213, 889-94
Shin HJ, Ko ES, Yi A (2015). Breast cancer screening in Korean woman with dense breast tissue. J Korean Soc Radiol, 73, $279-86$

Stavros AT, Thickman D, Rapp CL, et al (1995). Solid breast nodules: use of sonography to distinguish between benign and malignant lesions. Radiology, 196, 123-34

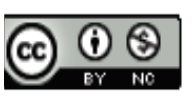

This work is licensed under a Creative Commons AttributionNon Commercial 4.0 International License. 